\title{
André Gide en Égypte : l'inachèvement et la créativité
}

Elizabeth GEARY KEOHANE, Université de Toronto à Scarborough

\section{Introduction}

André Gide a passé presque deux mois en Égypte en 1939 et en a remporté un carnet de voyage qui présente l'inachèvement non seulement comme thème principal, mais aussi comme source d'inspiration, quelque chose qui fait avancer le récit et propose une façon d'inscrire dans ses écrits les problèmes de créativité qui se présentent vers la fin de sa vie. Carnets d'Égypte est un ouvrage qui représente beaucoup plus ouvertement l'activité pédérastique de son auteur que ses écrits précédents ; quelques critiques l'ont déjà considéré dans le contexte des explorations sexuelles de Gide. ${ }^{1}$ En examinant l'ouvrage inachevé d'Edward Said, On Late Style, publié en 2006, nous allons situer ce court texte de Gide dans un contexte plus large - celui du rapport entre la créativité et le spectre de la mort - tout en réaffirmant la place importante que cet ouvrage mérite dans l'œuvre gidienne. Bien que Carnets d'Égypte représente un des derniers ouvrages de Gide (le livre a été publié en 1954, trois ans après sa mort), il ne s'agit pas d'une tentative d'arriver à la complétude ni de parachever une œuvre à la fois prolifique et variée, mais plutôt d'une création basée sur l'inachèvement. Dans ce contexte, l'inachèvement se rapporte à ce que Gide a choisi de ne pas « terminer » ou même de ne pas «finaliser »-l'expérience du voyage lui-même et surtout le processus d'écriture au travers duquel l'écrivain essaie de représenter cette expérience. «Late style » est une notion que développe Said à partir de l'idée de Spätstil chez Adorno. Ce terme décrit une mentalité qui se manifeste chez certains auteurs et chez certains compositeurs qui se trouvent devant la mort, que cela soit à l'âge mûr ou à cause d'une grave maladie. Said précise qu'il s'agit de découvrir ce qu'il appelle « un nouvel idiome » (6) pour ceux qui approchent la fin naturelle de leur productivité. Dans son texte, Said soulève certaines questions afin d'établir sa propre version du concept :

Each of us can readily supply evidence of how it is that late works crown a lifetime of aesthetic endeavour. [...] But what of artistic lateness not as harmony and resolution but as intransigence, difficulty, and unresolved contradiction? What if age and ill health don't produce the serenity of « ripeness is all »? (7)

Le but de Said dans cet ouvrage est d'explorer ce qu'il appelle «the experience of late style that involves a non-harmonious, nonserene tension and above all, a sort of deliberately unproductive productiveness going against [...]»(7). Il précise que : «Late style is what 
happens if art does not abdicate its rights in favor of reality » (9). Comme son appellation le suggère, « late style » remet en question la temporalité. "Late style » porte souvent sur l'anachronique et le rétrospectif ; c'est une façon de créer qui peut consister à mettre le temps hors de ses gonds, pour faire appel à la réplique d'Hamlet. Pour Said, « Late style is in, but oddly apart from the present » (24). Un auteur peut donc faire avancer son texte en regardant en arrière, comme le fait Gide. Stathis Gourgouris parle aussi du jeu temporel au cœur de « late style » en remarquant que «Late style is precisely the form that defies the infirmities of the present, as well as the palliatives of the past, in order to seek out [a] future... » (45). Examinant des ouvrages qu'écrit Ibsen à la fin de sa carrière, Said constate : «Far from resolution [...] Ibsen's last plays suggest an angry and disturbed artist for whom the medium of drama provides an occasion to stir up more anxiety, tamper irrevocably with the possibility of closure, and leave the audience more perplexed and unsettled than before » (7). La complétude n'est donc plus le but qu'il faut atteindre en écrivant à la fin d'une longue carrière littéraire ; l'idée de finaliser ou couronner son œuvre n'est plus un moteur de création pour l'écrivain, mais devient plutôt une platitude à éviter ou même un obstacle à contourner dans sa production littéraire.

Le choix de se concentrer sur l'inachèvement et non sur la complétude dans un tel cas signifie donc une résistance à la fois discordante et intransigeante chez l'écrivain. Toutefois, cette résistance pourrait être productive. Pour Said, la productivité fait partie du concept de « late style »; dans les ouvrages ainsi identifiés, il souligne la nécessité d'avoir un élément constructif avant tout (18). Nous allons donc voir que cette résistance de la part de Gide arrive à faire avancer le processus d'écriture justement parce que la manifestation de cette attitude comprend le contenu de son carnet. Dans un article qui explore la notion de clore un récit de voyage dans la littérature de voyage contemporaine, Jan Borm remarque que «l'on observe un nombre important de récits contemporains dont la fin reste plus "ouverte" dans la mesure où elle ne prend pas la forme d'un itinéraire bouclé » (35). La résistance que démontre Gide est peut-être un signe annonciateur de cette « ouverture », voire de cette inventivité, en ce qui concerne l'idée d'achever un carnet de route. En fait, Said mentionne la possibilité d'un tel « paradoxe » (17) dans le context de « late style », c'est-à-dire, « how essentially unrepeatable, uniquely articulated aesthetic works written not at the beginning but at the end of a career can nevertheless have an influence on what comes after them » (17-18). Pour Gide, il y a toujours un retour, certes, alors la notion de clore le voyage, c'est-à-dire, le terminer en rentrant chez soi, constitue, à première vue, une partie intégrante de (ou même un point de départ pour) ses récits de voyage. En même temps, il faut avouer que Gide ne commence jamais un voyage dans le but de trouver une nouvelle structure pour le récit de voyage qu'il compte écrire ; il s'agit 
plutôt d'adapter son texte en fonction des multiples difficultés qui se présentent lors de - et même avant - son périple. Si le voyage continue à l'habiter après son retour, par exemple, le texte peut aussi continuer à évoluer, comme nous allons le voir. En Égypte, Gide trouve la possibilité de faire avancer son récit au travers d'une résistance à l'imposition de la complétude comme réaction traditionnelle face à la mortalité. La possibilité de résister à cette norme représente pour lui un parcours aussi inspirateur que celui constitué par le déplacement physique, c'est-à-dire, le voyage lui-même. Bien que des fois accablé par l'ennui en Égypte « [j] veux ignorer qu'il y a 'des choses à voir' », écrit-il (546) - l'idée de l'inachèvement n'est pas quelque chose de négatif pour Gide parce que sa focalisation sur l'inachèvement dans ce récit de voyage (à savoir, la double difficulté de profiter d'un voyage à son âge et de continuer son travail d'écrivain lors du périple) est exactement ce qui remplit les pages de son journal.

\section{Late style face au désir}

Il est généralement admis que Carnets d'Égypte est le dernier récit de voyage de Gide, qui en a publié plusieurs de son vivant, notamment Voyage au Congo et Retour de l'U.R.S.S. et les textes qui les accompagnent, Le retour du Tchad et Retouches à mon Retour de l'U.R.S.S., respectivement. Le Congo et la Russie représentent de grands voyages qui ont marqué sa vie et ont produit des publications séparées de son Journal. Cependant, l'ouvrage Carnets d'Égypte a été intégré dans le Journal, ce qui nous révèle que ceci n'est pas un voyage comme les autres périples formateurs qu'il a entrepris dans sa vie. Tout d'abord, la représentation du voyage en Égypte ne se sépare pas facilement des autres réflexions personnelles de Gide qui se trouvent dans le Journal. Carnets d'Égypte est un texte remarquable non seulement dû à la mise en lumière de sa propre construction (que nous allons examiner plus tard), mais aussi parce que Gide y décrit en détail les vicissitudes de ses explorations sexuelles. Il est vrai que son voyage en Égypte n'a pas les mêmes ramifications politiques que son séjour au Congo ou en U.R.S.S., mais le récit de voyage qu'il y écrit présente une approche tellement honnête (vis-à-vis de ses goûts et de ses désirs) que Gide se montre tout de même conscient des problèmes que cela pourrait créer pour lui en France : «[1]es pages que j'écrivais hier indigneront certains », écritil (557). Faute d'amis avec qui partager ses expériences sur le vif, Gide, comme le souligne Naomi Segal, « is making the text his cruising companion ....» (148) (Nous pouvons aussi souligner le double sens de «cruising » ici - l'idée de voyager ainsi que celle de partir à la recherche d'une rencontre sexuelle.) En effet, Gide voyage avec le texte. Le texte n'est pas quelque chose qu'il produira peut-être s'il trouve suffisamment d'inspiration en Égypte ; le texte est une certitude, ce qui découlera de son périple, quoi qu'il arrive. Voyageant sans amis 
proches, mais toujours à la recherche des aventures intimes, Gide traite le texte de confident ainsi que de compagnon de voyage ; l'auteur est toujours prêt à y décrire ses désirs et ses actes transgressifs d'une manière extrêmement franche. Sans la présence d'un intermédiaire, Gide trouve que le texte s'offre comme réceptacle pour toutes ses expériences, même les plus explicites (rappelons que, pour lui, une telle honnêteté à l'écrit n'était pas possible du vivant de sa femme Madeleine $)^{2}$. Pour la plupart, ses aventures n'aboutissent à rien - des sourires et des caresses à la place d'une relation plus intime - une autre sorte d'inachèvement intentionnel qui est évident dans le texte. Au début de son voyage, Gide a du mal à trouver en Égypte une beauté masculine qui le fascine. Mais dès qu'il rencontre de jeunes hommes prêts à faire une transaction sexuelle, pour ainsi dire, il se contente d'échanger seulement quelques caresses avec eux : «Non, je n'ai plus grand désir de forniquer [...] Mais j'ai besoin de savoir que, si je voulais, je pourrais ; comprenez-vous cela ? »(547). Ce jeu fort-da fait penser à l'idée semblable de vouloir tenir à distance son carnet et d'y faire appel - bien que Gide se plaigne souvent du journal qu'il est censé écrire sur place, il prend plaisir à le conserver comme réceptacle de ses impressions et de ses frustrations lors du périple. Une telle contradiction interne constitue encore une autre facette du concept de « late style ». Said souligne que certains auteurs qui font preuve de «late style » dans leurs œuvres ne cherchent pas à résoudre la contradiction interne entre le désenchantement et le plaisir :

[Late style] has the power to render disenchantment and pleasure without resolving the contradiction between them. What holds them in tension, as equal forces straining in opposite directions, is the artist's mature subjectivity, stripped of hubris and pomposity, unashamed either of its fallibility or of the modest assurance it has gained as a result of age and exile. (148)

Le désenchantement et le plaisir représentent les deux extrêmes de l'expérience de Gide en Égypte : la difficulté de profiter du voyage afin de pouvoir écrire sur le pays visité, mais la possibilité omniprésente d'y trouver la satisfaction physique. L'auteur, expérimenté grâce à son âge et à son exil volontaire et temporaire (1'auto-exil, sous forme de voyage de découverte, étant une des raisons pour lesquelles il voyage autant), comprend que ces deux extrêmes peuvent coexister ; voilà pourquoi il creuse ses expériences dans son journal de voyage afin de le transformer en un espace dans lequel il n'est pas question de résoudre les différences. Au lieu d'atteindre un état d'harmonie dans ce texte, Gide tente d'en tirer la valeur artistique propre à ces contradictions. Cette approche toujours ambivalente crée donc une sorte de polyvalence qui l'aide à enrichir son récit. 


\section{La construction et la rédaction du journal de voyage}

Nous regarderons maintenant plus en détail la façon dont Gide souligne la construction de Carnets d'Égypte comme partie intégrante du texte. Tout d'abord, le titre semble nous indiquer qu'il s'agit tout simplement d'un autre carnet de route par le grand écrivain-voyageur français du vingtième siècle. Pourtant, dès les premières lignes de Carnets d'Égypte, la construction du texte est mise en évidence, devenant ainsi quelque chose d'encore plus important que la description du pays visité : «J'écris ceci pour dérouiller ma plume et m’entraîner ; et aussi parce que je me sens, vis-à-vis du Caire, dans un état d'incuriosité totale » (543). L'idée du carnet promis - celle du texte «à venir »- le force à écrire. Même si écrire ce texte représente une lutte pour lui, il choisit tout de même de le faire et essaie d'y intégrer cette difficulté créative. Le manque d'inspiration dont il fait preuve, est alors surmonté par sa détermination de créer, d'écrire quelque chose, de faire avancer son journal - de s'entraîner, pour reprendre son propre terme, un verbe qui fait penser à un apprentissage continuel, certes, mais qui semble faire référence aussi au mouvement. La lutte et l'échec se trouvent donc inscrits dans le texte. Pour nous servir de la belle image qu'utilise Adorno pour décrire la présence du « late style » chez Beethoven, le texte de Gide met en valeur le vide qui l'étaye, ou « turns its emptiness outward » (11). Nous sommes donc témoins de ce que Gourgouris appelle «internal tearing » (38) dans son analyse du concept de « late style » chez Said. Ce dernier précise que « late style » est à la fois objectif et subjectif pour Adorno, qui parle de nouveau du cas de Beethoven : «Objective is the fractured landscape, subjective the light in which - alone - it glows into life. He does not bring about their harmonious synthesis. As the power of dissociation, he tears them apart in time, in order perhaps, to preserve them for the eternal. In the history of art, late works are the catastrophes » (12). Encore une fois, nous voyons cette intransigeance productive, c'està-dire « a sort of deliberately unproductive productiveness going against » (7). Ainsi le chaos, la catastrophe et la contradiction sont-ils favorisés ; pour l'écrivain, ainsi que pour le compositeur, l'harmonie n'est plus le but à atteindre. En outre, la détermination de Gide de produire un texte, même à partir de sa frustration créative, montre une autre facette de sa résistance ; il réagit contre les conditions de son âge et de sa maturité, bien sûr, mais aussi contre les conditions d'un voyage qui n'arrive pas à l'éblouir. Son carnet présente un champ de bataille, pour ainsi dire, auquel nous ne nous attendions pas ; ces références à la difficulté d'écrire dès le début de ce texte ne sont pas nécessairement typiques d'un grand homme de lettres qui a autant publié dans sa vie, ni de quelqu'un de curieux qui se trouve en Égypte pour la première fois. La mise en lumière de cette difficulté montre qu'il va à l'encontre d'une telle image de l'écrivain à l'âge mûr, qui serait résolu à réaffirmer l'importance ainsi que la 
cohérence de son œuvre. D'autres remarques franches au sujet de la création littéraire se trouvent ailleurs dans le texte. En effet, nous voyons que l'idée d'« écrire » est, pour Gide, toujours une question de $s$ 'entraîner à écrire :

Je rentre vider malle et valise - je m'installe et me persuade que je vais pouvoir travailler. (547)

Rien noté hier. Ce carnet ne m'intéresse pas du tout. (549)

Plus aucun désir de tenir à jour ce carnet : le charme est rompu. (558)

Je me force pour tenir à jour ce carnet, mais ne trouve aucun plaisir à y rien écrire. (564)

La notion de faire référence à la construction de l'ouvrage dans le texte lui-même montre aussi le désir de Gide de ne pas fixer son expérience du voyage. Comme lecteurs, nous avons l'impression de veiller sur un texte en train de se dessiner. D'ailleurs, en gardant le format à la fois fragmentaire et unitaire du journal, Gide peut toujours alimenter ses notations d'autres détails dans l'avenir. L'ouvrage restera donc un texte « en construction » et pourrait être adapté à n'importe quel moment, comme Gide l'a déjà fait pour ses textes sur l'Afrique équatoriale (en ajoutant des notes de bas de page ainsi que des appendices après son retour) et sur l'U.R.S.S. (en écrivant Retouches à mon Retour de 1'U.R.S.S.). Ceux qui analysent ces récits de voyage en particulier, peuvent souligner que les destinations dont il est question ici, des lieux qui sont eux-mêmes en pleine évolution, méritent ce genre de traitement provisoire pour ainsi dire ${ }^{3}$. Mais, comme destination, l'Égypte ne se transforme pas à la même vitesse. En fait, Gide souligne à quel point cette destination est « momifiée », pour ainsi dire : «L'Égypte... engainée dans ses bandelettes », écrit-il, avant de faire appel à la conclusion d'un livre sur l'histoire du pays écrit par l'égyptologue Alexandre Moret, dans laquelle l'auteur précise que « l'émancipation qui oppose aux exigences de la société les droits de l'individu [...] ne s'est jamais réalisée en Égypte » (558). Pourquoi donc ce pays mérite-t-il une approche similaire dans le récit de voyage qui découle de ce périple ? Il importe de mentionner les autres projets littéraires sur lesquels Gide travaille lors de son voyage. Il est en train de corriger les épreuves de son Journal et essaie aussi de terminer un texte sur sa femme décédée, un ouvrage qui sera intitulé Et Nunc Manet in Te. Se consacrer au voyage n'est donc pas la raison d'être de ce périple ; nous voyons que Gide tient l'Égypte à distance et se retire du pays, pour ainsi dire, afin de se concentrer sur la création littéraire. D'ailleurs, Gide s'avère moins passionné par ce pays en particulier : «Ce grouillement de population avec qui je ne me sentais rien de commun m'a plongé dans un marasme sans nom » (544). Gide écrit donc un récit de voyage dans lequel le voyage lui-même n'est pas le moteur principal qui motive la progression du texte ; tout simplement, l'auteur se rend ailleurs pour travailler, pour écrire : «Sans doute pourrai-je 
retrouver ici, loin de tout, un état propice au travail [...]», insiste-t-il (546). Pour Joseph Boone, Carnets d'Égypte est une exception dans l'œuvre de Gide, « a stripped-down, matter-of-fact diary without the literary embellishments and experimental techniques that make the Algerian entries [Amyntas] such an evocative investigation of self and otherness deployed for simultaneously modernist and homoerotic ends [...]»(224-225). Cependant, c'est exactement cette apparente qualité dépouillée qui est vraiment innovatrice dans le contexte des récits de voyage de Gide ; loin de valoriser l'exotique comme Amyntas ou même Voyage au Congo, Carnets d'Égypte semble rejeter une exploration de l'autre afin que Gide puisse mieux se concentrer sur lui-même : ses désirs, ses projets littéraires. Le texte nous fournit un regard encore plus introspectif sur l'écrivain - c'est un journal de voyage qui s'intègre bien dans le Journal, puisqu'il s'agit d'un journal intime plutôt que d'un journal sur l'extérieur. Gide explique plus en détail ce mouvement vers l'intérieur, au détriment d'explorer tout ce qui est extérieur, dans son dernier texte, Ainsi soit-il ou Les jeux sont faits :

Je n'avais pas pris mes dispositions pour vivre aussi vieux. À partir d'un certain âge, il m'a semblé que je quittais mon rôle. Mon optimisme devenait guindé, ou bien il battait en retraite. Il me fallait bien reconnaître que l'aspect du monde ne le justifiait guère et que je ne parvenais à le maintenir qu'en cessant de regarder ailleurs qu'en moi. (182)

Dans Carnets d'Égypte, Gide nous montre à plusieurs reprises que son état introspectif n'est pas nécessairement une séparation totale du monde extérieur, mais une façon de se concentrer sur le «moi » plutôt que sur l'autre : «Bien décidé à simplement me laisser vivre, tout aujourd'hui, comme un palmier. Je veux ignorer qu'il y a 'des choses à voir', à Louxor et aux environs » (546) révèle-t-il, parmi d'autres exemples.

\section{Voyageant à l'âge mûr}

Lié à l'exploration de la sexualité, le rapport qui existe entre la jeunesse et l'âge mûr, comprend un autre aspect fondamental de Carnets d'Égypte. Ce rapport est, bien sûr, au cour d'autres récits de voyage écrits par Gide, mais nous allons voir que Gide le traite de façon différente dans le carnet égyptien. Pour lui, l'idée de voyager au Congo ou en U.R.S.S. présentait aussi la possibilité de rajeunir. Dans une lettre à Marc Allégret, qui l'accompagnerait ensuite en Afrique équatoriale, Gide explique que « La pensée de ce voyage me fait tourner la bobine. Je n'arrive pas à me persuader que c'est moi qui vais réaliser cela ! Qu'il fait beau, déjà ! Chaque jour j'attends la catastrophe qui va rendre cela impossible [...] Ah ! vieux, je vais tellement mieux, je suis tellement plus jeune et plus avide qu'il y a vingt ans ! »(608-609). D'ailleurs, lors d'un entretien avec Jean Amrouche en 1949 (publié dans le livre d'Éric Marty), Gide utilise 
aussi les images frappantes de trouver « un bain de nouveauté, [...] un bain de jouvence » (263) comme raisons pour lesquelles il a entrepris son voyage au Congo. Quant à l'U.R.S.S., Gide voit le renouvellement de l'humanité comme partie intégrante de la société soviétique et se sert de maintes images de la jeunesse dans les deux textes qui représentent ce voyage pour souligner cette conviction. De plus, même avant son départ pour le Congo, Gide parle de l'importance de la jeunesse comme mode d'instruction pour les aînés dans un autre texte de l'entre-deuxguerres, Incidences :

On a dit que je cours après ma jeunesse. Il est vrai. Et pas seulement après la mienne. Plus encore que la beauté, la jeunesse m'attire, et d'un irrésistible attrait. Je crois que la vérité est en elle ; je crois qu'elle a toujours raison contre nous. Je crois que loin de chercher à l'instruire, c'est d'elle que nous, les aînés, devons chercher instruction. (94)

Dans Carnets d'Égypte, cependant, bien que la jeunesse soit d'une importance fondamentale pour l'écrivain, le spectre de la mort hante le texte. Gide parle de sa propre mort - « la dépouille que je vais bientôt devoir au tombeau » (557) - et se met à imaginer son meurtre aux mains des jeunes hommes qu'il rencontre. Plus tard, il y a un incident bizarre où l'écrivain et quelques camarades font un pique-nique dans un cimetière - « [U]n cimetière arabe offre l'aspect presque riant de ses tombes blanches [...]» (571) - tout en regardant un enterrement arabe qui s'y déroule. Said souligne ce que dit Adorno à l'égard de Beethoven : « late style does not admit the definitive cadences of death ; instead, death appears in a refracted mode, as irony [...] » (24). Pour Gide, la possibilité de représenter la mort dans ce texte arrive tout de même à tenir ce concept à une certaine distance. D'ailleurs, l'idée de se concentrer plutôt sur la jeunesse lui permet avant tout «de n'être pas trop mélancolique ; juste un peu, car la seule jeunesse qu'aucun printemps ne ramènera, c'est la mienne. Puissé-je avoir laissé d'elle quelque chaleur en mes écrits ! » (537). Il se peut que Gide cherche à laisser « quelque chaleur » de sa jeunesse dans son œuvre justement pour atténuer - ou continuer à défier - la mort qui est aussi présente, bien que de façon réfractée ou déformée, dans ce qu'il écrit.

Après une brève discussion de Delphes (ce qui nous révèle le retour soudain de l'auteur en Grèce, une partie du voyage qui n'est pas décrite dans le texte), le carnet s'achève brusquement en citant, sans aucune introduction, le philosophe Francis Bacon : «Natura non imperat nisi parendo » (574). Cette maxime nous indique que la nature peut être maitrisée si et seulement si on y obéit. Elle met l'accent sur la puissance de la nature ; dans le cas de Gide, cela fait penser à la mort inévitable à laquelle il doit faire face, mais aussi contre laquelle il réagit au travers de son journal. Gide place cette citation, non sans ironie, à la fin de son texte, déconnecté du texte principal, dans le dessein de suggérer que c'est la mort qui va tout finaliser, 
au lieu de l'écrivain lui-même. Cette citation constitue un arrêt net, certes ; les mots en italique isolés au centre de la page font penser à une épitaphe. Mais, en même temps, cette maxime suscite un nouveau débat pour le lecteur : qu'est-ce que cela veut dire, terminer un voyage ? Si l'idée de terminer un voyage est toujours remise en question, comment finaliser sa représentation? Il importe de souligner que, pour Gide, même depuis un très jeune âge, à l'écrit, le voyage risque de ne pas s'accomplir, de ne pas se terminer. Prenons, par exemple, la représentation du voyage dans ses écrits fin-de-siècle : notamment, au cœur du Voyage d'Urien (1893), de Paludes (1895) et d'El Hadj (1896) se trouve un périple inachevé et souvent peu satisfaisant pour le voyageur en question. Comme pour Des Esseintes, le personnage principal dans le roman contemporain de Joris-Karl Huysmans, À rebours (1884), un retour ne garantit pas toujours que le voyage ait eu lieu. D'ailleurs, le vocabulaire qu'emploie Gide dans une lettre de 1895, indique que la linéarité n'est pas quelque chose qui le préoccupe quand il considère l'idée de la progression. Écrivant à Eugène Rouart, Gide parle de l'extase du voyage :

[J]e crois que la route que je suis est ma route; et que je la suis comme il faut [...] Durant de longs mois je n'ai rien étudié que la vie ; je ne comprends plus aussi bien les livres ; ils ne m'ont plus l'air tout comme avant ; - et pourtant je me sens l'intelligence admirablement élargie ; l'égarement de ma conduite à présent vient comme une suite naturelle de ce débordement de ma pensée - de cette évasion de ma pensée. N'étant plus restreinte elle n'est plus retenue [...] Ô Splendeurs ! ô délices de l'exubérance ! mon cerveau m'est une forêt de l'Inde aux végétations embrouillées et mon émotion y circule dans l'enchevêtrement des lianes. (t. I, 263)

Le motif du voyage inachevé dans ses écrits de la fin-de-siècle ainsi que l'introduction d'un vocabulaire de non-linéarité - «égarement », « évasion » et « enchevêtrement »- dans cette lettre contemporaine sur le voyage nous indiquent que la progression n'est jamais facile chez Gide, peu importe son âge. Mais la résistance aux multiples facettes qui met le temps hors de ses gonds dans son dernier récit de voyage, Carnets d'Égypte, est d'une certaine manière différente. Cette résistance intransigeante constitue un nouveau départ pour Gide et suscite une problématique qui était auparavant sous-estimée dans son œuvre : comment faire face à la mort imminente dans ce que l'on écrit? Le dernier texte de Gide, Ainsi soit-il ou Les jeux sont faits, sur lequel il travaillait jusqu'à son décès, continue à explorer la possibilité de résister à l'idée que la mort peut guider un auteur vers l'achèvement de son œuvre. Nous revoyons dans ce texte les mêmes motivations et les mêmes réflexes déjà présents dans Carnets d'Égypte : le refus, la défiance, l'intransigeance, la contradiction. À titre d'exemple, voici un extrait des dernières lignes d'Ainsi soit-il, qui ont été écrites six jours avant la mort de l'auteur en février 1951 : 
Non ! Je ne puis affirmer qu'avec la fin de ce cahier, tout sera clos ; que c'en sera fait. Peut-être aurai-je le désir de rajouter encore quelque chose. De rajouter je ne sais quoi. De rajouter. Peut-être. Au dernier instant, de rajouter encore quelque chose. J'ai sommeil, il est vrai. Mais je n'ai pas envie de dormir. Il me semble que je pourrais être encore plus fatigué. Il est je ne sais quelle heure de la nuit, ou du matin... Ai-je encore quelque chose à dire ? Encore à dire je ne sais quoi. (197-198)

Dans ces lignes émouvantes, la résistance à la mort, ou à la fin définitive, se présente de nouveau. La seule façon de repousser cette affreuse finalité est de continuer à écrire, même si c'est seulement la répétition des mots qui fait avancer sa plume. Même si le double titre de ce livre semble faire référence à son acceptation de la fin imminente, nous voyons que le contenu de ce dernier ouvrage continue à mettre en évidence les éléments clés d'un « late style » dont Carnets d'Égypte a déjà fait preuve.

\section{Conclusion : « Laissons à faire à la mort »}

Pour conclure, nous avons montré que Carnets d'Égypte pourrait être considéré comme un ouvrage qui montre chez Gide ce que Said, d'après Adorno, nomme « late style ». Gide, en se focalisant sur l'inachèvement comme thème et moteur de création, arrive à faire avancer son récit de voyage tout en y remettant en question les notions de progression et de complétude. Le but qu'il importe d'atteindre comme écrivain, n'est donc pas de finaliser un ouvrage, ni de parachever son œuvre à la fin de sa carrière, car l'idée d'inscrire une attitude de résistance dans ses écrits offre un chemin beaucoup plus enrichissant et revigorant pour l'écrivain face à sa propre mortalité. Comme Gide le précise dans Carnets d'Égypte, «Laissons à faire à la mort ; à quoi bon aider à son jeu ? » (557). Comme nous l'avons vu, au lieu d'aider ce « jeu » propre à la mort, l'écrivain peut essayer de transformer son récit en un espace ludique, dans lequel il peut commencer à interroger le concept de clore un texte, ou la notion d'achever son œuvre. Il peut se servir d'un tel espace afin de résister à la mort inévitable, justement en s'inspirant de cette apparente limite à la créativité.

\section{Ouvrages cités}

Boone, Joseph. The Homoerotics of Orientalism. New York : Columbia UP, 2014.

Borm, Jan. «Clore un récit de voyage. »Études britanniques contemporaines 10 (1996) : 27 35.

Gide, André. Ainsi soit-il ou Les jeux sont faits. Paris : Gallimard, 1952. 
---. Carnets d'Égypte. Voyage au Congo/Le Retour du Tchad/Retour de l'U.R.S.S./Retouches à mon Retour de l'U.R.S.S./Carnets d'Égypte. Paris : Gallimard «Biblos », 1993. 541574.

---. Incidences. Paris : Gallimard, 1946 [1924].

--- et Marc Allégret. Correspondance 1917-1949. Dir. Jean Claude et Pierre Masson. Paris : Gallimard, 2005.

--- et Eugène Rouart. Correspondance André Gide-Eugène Rouart. 2 vol. Dir. David Walker. Lyon : PU de Lyon, 2006.

Gourgouris, Stathis. «The Late Style of Edward Said». Alif : Journal of Comparative Poetics 25 (2005) : 37-45.

Marty, Éric. André Gide - Qui êtes-vous ? Lyon : La Manufacture, 1987.

Said, Edward. On Late Style. Londres : Bloomsbury, 2006.

Segal, Naomi. «Gide in Egypt 1939 ». Cultural Encounters : European Travel Writing in the 1930s. Dir. Charles Burdett et Derek Duncan. New York / Oxford : Berghahn, 2002. 143-155.

\section{NOTES}

1. Pour une analyse plus approfondie des explorations sexuelles de Gide en Égypte, voir l'essai de Naomi Segal et le livre récent de Joseph Boone.

2. Nous ne pouvons pas sous-estimer l'effet que la mort de Madeleine a eu sur Gide. Comme le dit l'auteur dans Ainsi soit-il ou Les jeux sont faits : «Je pèse chaque mot que j'écris ; m'en voudrais d'outrer ma pensée. Somme toute, la partie que je jouais, je l'ai gagnée. Mais j'ai cessé de m'y intéresser vraiment depuis que Em. [Madeleine] m'a quitté. Depuis, il me semble souvent que je n'ai plus fait que semblant de vivre : elle était ma réalité. Peu importe si je ne me fais pas comprendre » (15).

3. Dans ma thèse de doctorat sur Gide, j'ai souligné, dans le contexte de Retour de l'U.R.S.S., l'impact d'un lieu « en construction» sur le journal de voyage ainsi que les difficultés qui se présentent pour l'auteur qui essaie de décrire une destination en période de transition. 